\title{
Experimental research of cavity geometry behind high-speed bodies in water
}

\author{
Alexander Ischenko ${ }^{1}$, Victor Burkin ${ }^{1}$, Alexey Diachkovskiy ${ }^{1}$, Anton Sammel ${ }^{1, *}$, and Andrey \\ Chupashev $^{1}$ \\ ${ }^{1}$ National Research Tomsk State University, 36, Lenin Ave., Tomsk, 634050 Russia
}

\begin{abstract}
At high speed launching of bodies via smoothbore throwing facility into water, a stabilization of bodies was experimentally achieved due to periodical interactions of body contour with the cavity boundaries. If the body contours are not exceeding produced cavity boundary, drag force localized mostly at nose part of a body, which calls "cavitator". Based on this, it will be rational to take into consideration cavity shape when designing contours of bodies.
\end{abstract}

\section{Introduction}

The process of body movement in water medium is significantly different from a body movement in atmospheric gas. This difference is growing up when a body speed exceeds a hundred meters per second. The nasal part of a body breaks continuity of water medium and almost the entire body contour is moving inside the cavity with rarefied environment. The cavity contours are very elongated along throwing axis, and if a body contours are not exceeding cavity contours, the cavity starts to stabilize a body while it moves.

A detailed physical description and analysis of the cavitation development mechanical aspects, and in particular of the developed cavitation, are given by L. I. Sedov [1]. An invaluable contribution to the research of this phenomenon was made by a follower of L.I. Sedov - G.V. Logvinovich, who formulated the currently accepted "cavity expansion independence principle" for an ideal fluid, which consists in applying the energy conservation equation to each section of the cavity, which was published in his work [2].

Currently, a number of research groups are working in this direction, among which should be noted the studies of Ukraine [3] and the USA [4, 5, 6].

\section{Experiments description}

During an experimental testing of elongated bodies shape at hydroballistic complex of RIAMM TSU [7, 8], the geometry of contours of cavities around bodies was estimated. Results were being used for bodies shape improvements and for comparison of the mathematical model with experimental data in range $317 \ldots 404 \mathrm{~m} / \mathrm{s}$.

\footnotetext{
*Corresponding author: anton_sammel@mail.ru
} 
As bodies an axisymmetric duralumin products with elongated conical nasal part were used. At bottom part of the bodies, grooves were carved for master devices attaching (Fig. 1).

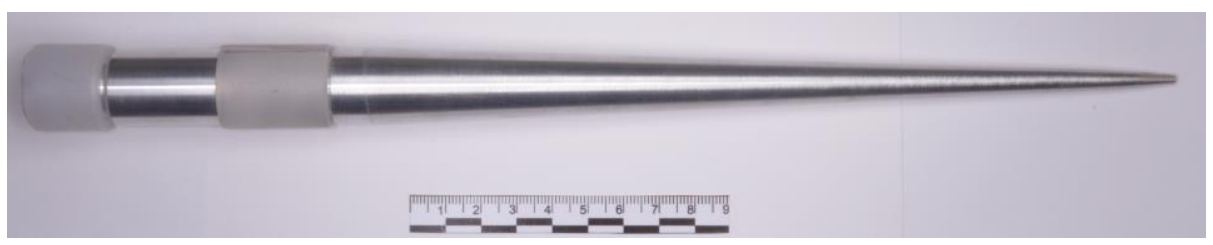

Fig. 1. Photo of elongated duralumin body

Fig. 2 shows a series of photographs. There are illustrated key phases of an underwater high-speed movement process: moving of a body inside the cavity $(a)$, maximum expansion of the cavity $(b)$, collapsing of the cavity $(c)$.
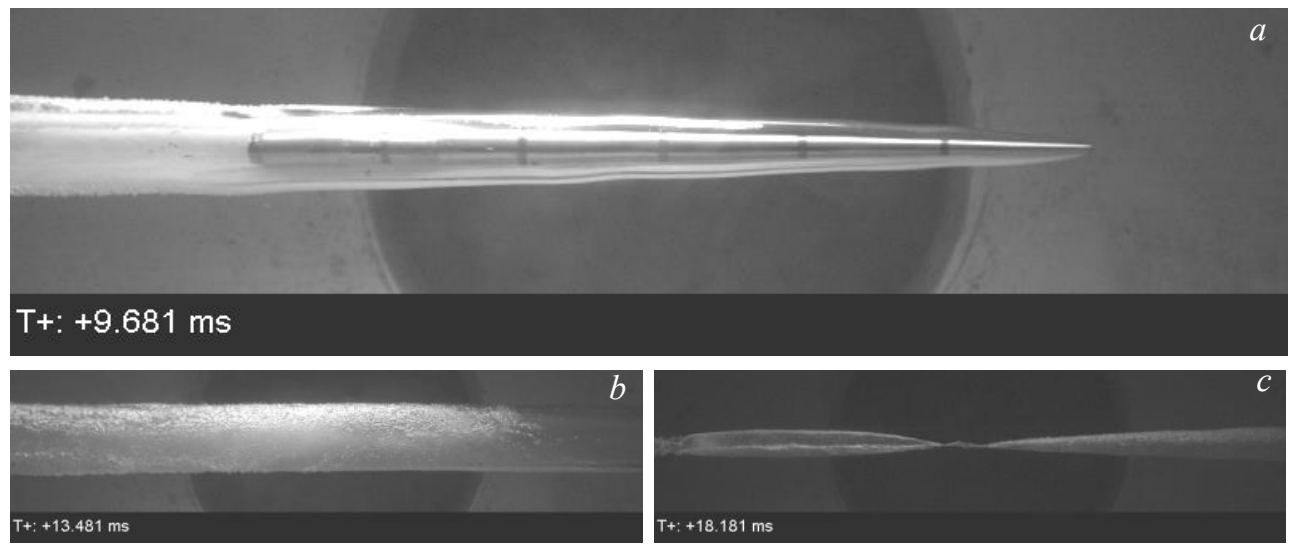

Fig. 2. Photos of underwater body movement process

According to [2], the closer body movement to straight and uniform, the more likely it can be expected that every section of the cavity expands almost independent on subsequent or previous body movement. Therefore, one unperturbed expanding section was selected, its diameter was measured at regular time intervals and its dynamics was projected to other cross-sections of the cavity in order to plot a characteristic profile of whole cavity behind a cavitator.

Profiles of cavities and displacement of bodies within experiments was measured via diffused light projection on high-speed camera image sensor at $20 \mathrm{kHz}$.

\section{Obtained results}

Fig. 3 shows results of measuring of undisturbed cavity section radius $R_{\mathrm{c}}$ relative cavitator radius $R_{0}$ versus time $t$ for different bodies speeds. 


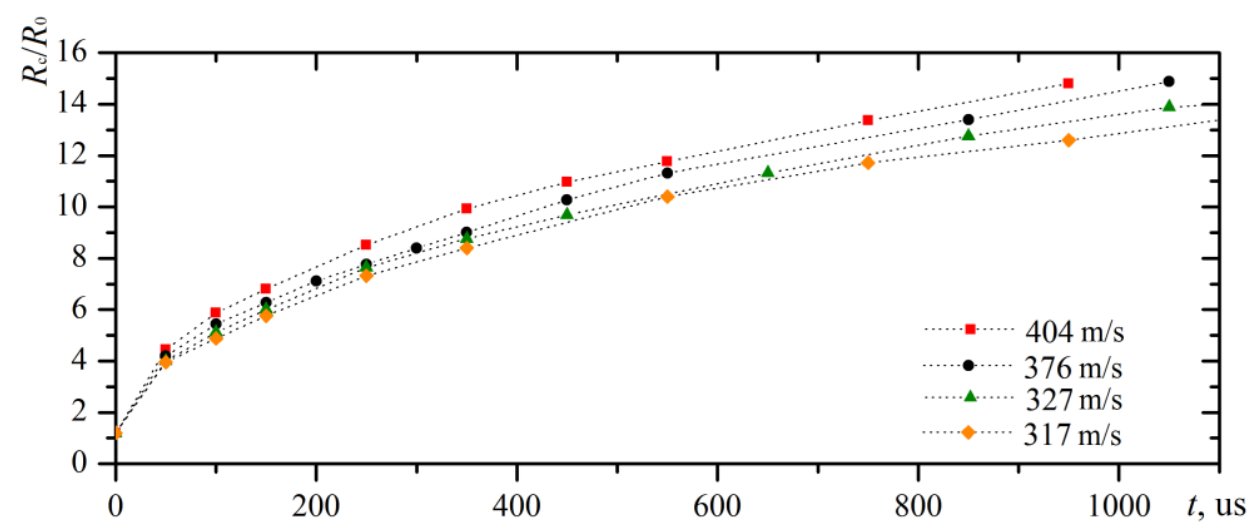

Fig. 3. Expansion of undisturbed cavity section versus time for different bodies speeds (initial period).

Fig. 3 shows speeds as estimations of each body average speed on an observation area (about $0.3 \mathrm{~m}$ ) obtained by high-speed imaging. There is a dependence of expansion rate on body speed.

Fig. 4 shows results of measurements of undisturbed cavity section radius throughout the period its lifetime. The dependence of the maximum cavity section diameter and the cavity section lifetime on the cavitator speed is noted.

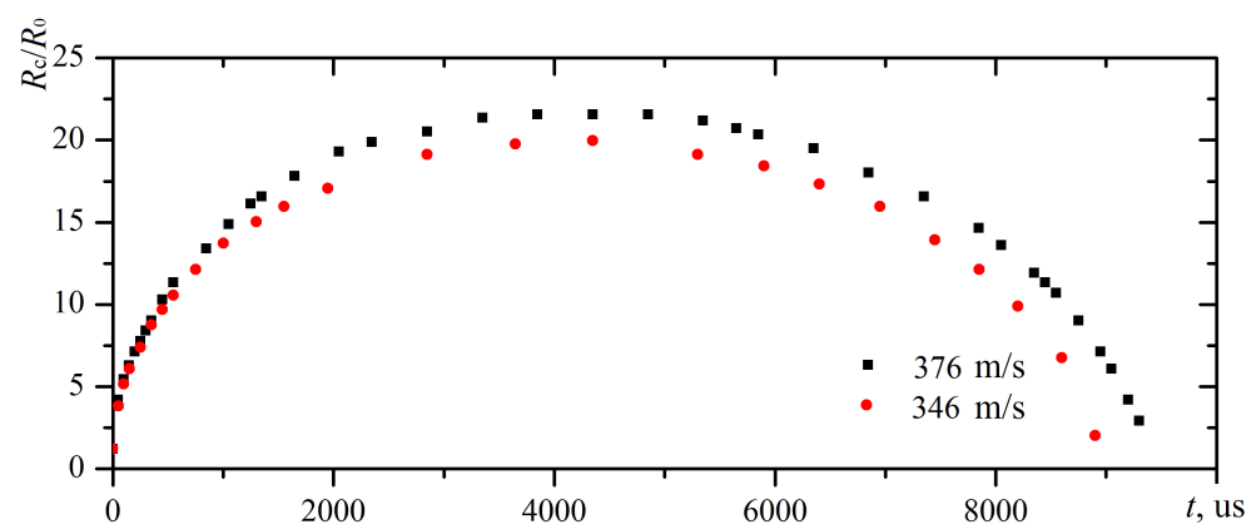

Fig. 4. Dependence of undisturbed cavity section radius on time for different body speeds (full period).

Fig. 5 shows experimental profiles of cavities at different speeds of bodies, they are plotted in cavitator related reference system. This way of data presentation under considered conditions indicates an absence of significant influence of body speed on cavity profile in cavitator related reference system. This is consistent with the data of other researchers at work [9]. 


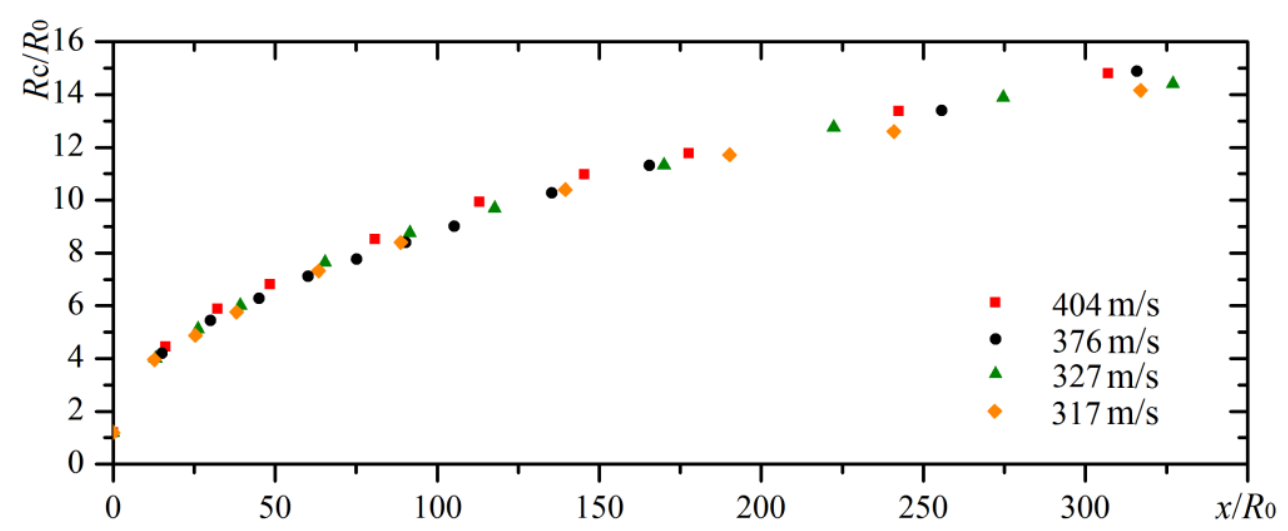

Fig. 5. Experimental profiles of cavities at different speeds of bodies.

Fig. 6 shows comparing the obtained data with results [10]. Data are shown as cavity midsection $R_{\mathrm{m}}$ to cavitator radius $R_{\mathrm{c}}$ ratio dependency on cavity number $\sigma$. The similar arrangement of the experimental dependences shown above should be noted.

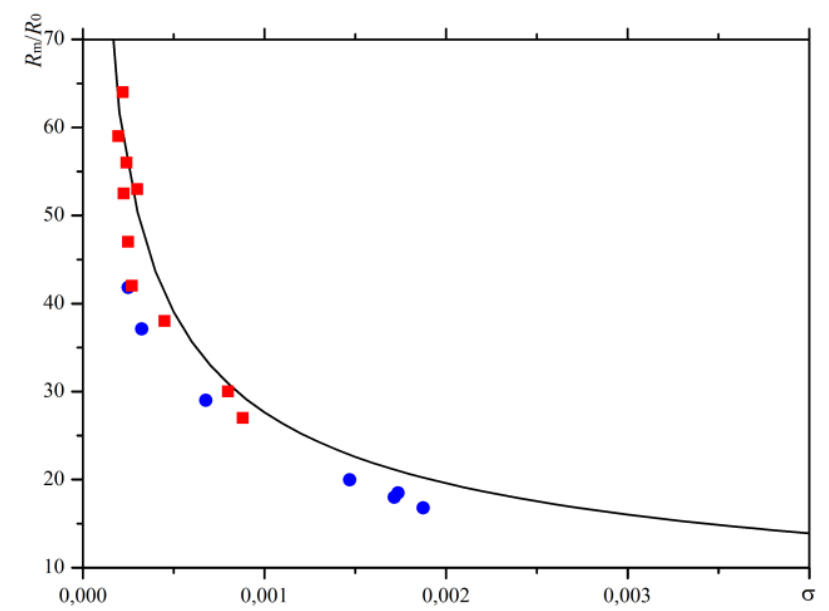

- - experiment [4]; — - calculation [4]; • - experiment RIAMM TSU;

Fig. 6. Cavity midsection $R_{\mathrm{m}}$ to cavitator radius $R_{\mathrm{c}}$ ratio dependencies on cavity number $\sigma$ is obtained in this work and the results published in [10].

\section{Conclusion}

Contours of cavities were formed by considered throwing bodies in water that were experimentally obtained. Results were compared with experimental data of other researchers and their theoretical calculations, and this comparison shows the close character of dependencies. These results let us predict forming cavity geometry, do refine body contours shape and lead ongoing development.

The research was supported by RSF (project No. 19-19-00233). 


\section{References}

1. L. I. Sedov, in Planar problems of hydrodynamics and aerodynamics ("Gostehizdat", 1951).

2. G. V. Logvinovich, in Hydrodynamics of free-boundary flows ("Naukova Dumka", 1969), p. 128.

3. Yu. Savchenko, V. Semenenko, S. Putilin, "Non-stationary processes in supercavitational motion of bodies", Applied hydromechanics. Vol. 1, 1 (73), pp. 7997.

4. T. T. Truscott, B. P. Epps and J. Belden, "Water entry of projectiles" Annual Review of Fluid Mechanics, 46, 355-378.

5. A. H. Techet and T. T. Truscott, "Water entry of spinning hydrophobic and hydrophilic spheres" J. of Fluids and Struct., 27(56), pp. 716-726.

6. Bodily KG. 2013. The water entry of slender axisymmetric bodies: forces, trajectories and acoustics. MS thesis. Brigham Young Univ., Provo, UT.

7. V. V. Burkin., A. N. Ischenko, I. V. Maistrenko, V. M. Fufachev, A. S. Diachkovskiy., V. A. Burakov, L. V. Korolkov, E. Y. Stepanov, A. V. Chupashev, K.S. Rogaev, A. Y. Sammel, A. D. Sidorov, RU Patent "Hydroballistic track", request № 2017135871 from 09.10.2017.

8. A. N. Ishchenko, V. V. Burkin, A. S. Diachkovskii, L. V. Korolkov, A. I. Zykova, A.V. Chupashev, "Visualization of high-speed interaction of bodies in water" in Proceedings of the 18th International Conference on the Methods of Aerophysical Research (2016) Vol. 1770, 030011 (2016).

9. G. V. Logvinovich, V. V. Serebryakov, "About determination of dimensions of the cavity in water behind a high speed cylindrical body" J. Tech. Phys., 83 (2013), pp. 15-20 (in Russian).

10. Y. D. Vlasenko, "Experimental investigation of supercavitation flow regimes at subsonic and transonic speeds" Fifth International Symposium on Cavitation. Osaka, Japan (Cav03-GS-6-006). 